\title{
Avaliação de modelos volumétricos para plantio comercial de Calophyllum brasiliense Cambess na região sul do estado do Tocantins
}

\author{
Marcos Cardoso Silva ${ }^{a^{*},}$ Suelen Fernanda Goergen ${ }^{\mathrm{a}}$, Maria Cristina Bueno Coelho ${ }^{\mathrm{a}}$, Marcos Giongo ${ }^{\mathrm{a}}$, \\ Mauro Luiz Erpen ${ }^{\mathrm{b}}$, André Ferreira dos Santos ${ }^{\mathrm{a}}$ \\ a Universidade Federal do Tocantins, Brasil \\ ${ }^{\mathrm{b}}$ Instituto Federal de Ciência e Tecnologia do Tocantins, Brasil \\ *Autor correspondente (markuz@uft.edu.br)
}

\section{N F O}

Keywords
guanandi
rigorous cubage
forest biometrics

Palavras-chaves

guanandi

cubagem rigorosa

biometria florestal

\begin{abstract}
A B S T R A C T
Evaluation of volumetric models for commercial planting of Calophyllum brasiliense Cambess in the southern region of Tocantins state.

Rigorous cubing is the measurement of various diameters along the tree trunk to obtain the true volume of a single tree. To estimate a volume for a given planting, volumetric equations are used, which generate the volume with the least error and closest to the actual volume. Thus, rigorous cubing by the Smalian method was performed on 46 trees, determined by diameter class, with a minimum adopted diameter of $2 \mathrm{~cm}$. Nine volumetric equations were tested, based on the following criteria: Adjusted coefficient of determination $\left(\mathrm{R}^{2} \mathrm{aj}\right)$, coefficient of variation in percentage $(\mathrm{CV} \%)$, and graphical analysis of residues. With the data analysis, the best fit model was Schumacher-Hall, presenting the following results: adjusted $\mathrm{R}^{2}=0.979, \mathrm{CV} \%$ of 2.24 , the graphical analysis of residual dispersion between the observed and estimated values showed that the function $\ln (\mathrm{v})=-15.0053+1.29298 . \ln (\mathrm{d})+1.266519 . \ln (\mathrm{h})$ presented adequate adjustment. With the selected equation, the volume was estimated at $6.22 \mathrm{~m}^{3} \cdot \mathrm{ha}^{-1}$ year, considering 1,250 trees per hectare at 7 years.
\end{abstract}




\section{INTRODUÇÃO}

O Calophyllum brasiliense Cambess pertencente à família botânica Clusiaceae, mais conhecido popularmente como guanandi ou landi. É uma espécie nativa do Brasil que vêm sendo plantada atualmente, pelas suas ótimas características silviculturais e pela excelente qualidade de madeira. $\mathrm{O}$ guanandi é plantado em países como a Costa Rica há mais de 15 anos onde apresentou crescimento satisfatório (Carvalho, 1996). No Brasil, é encontrada na Amazônia, no Cerrado e na Mata Atlântica, desde o estado do Amazonas até Santa Catarina. Na Amazônia, é frequente nas várzeas e igapós. No Cerrados habita as matas de galeria. A espécie pode alcançar em plantios comerciais altura entre 20 e 40 metros, com o diâmetro de 40 a $60 \mathrm{~cm}$, possui uma grande durabilidade e resistência, podendo ser cultivada desde solos com pouca fertilidade até solos alagados (Cidrão, 2012).

A espécie Calophyllum brasiliensis Cambess tem sido excelente opção para indústrias do setor florestal brasileiro. É a primeira madeira de lei do país (lei de 7 de janeiro de 1835), se adapta aos diversos tipos de clima e solo e pode ser encontrada de norte a sul do país. Possui excelentes características silviculturais, ótima qualidade da madeira e é muito utilizada para fabricação de móveis finos (Duffecky e Fossati, 2009). Possui características na sua madeira que se assemelha ao cedro e ao mogno e em alguns países da América Latina e Central vem substituindo estas espécies em grande escala (Cidrão, 2012).

Neste contexto o estado do Tocantins surge como alternativa para plantios comerciais de $\mathrm{Ca}$ lophyllum brasiliensis Cambess, pois, a produção sustentável e o crescimento do setor florestal nas últimas décadas fizeram com que o estado do Tocantins obtivesse considerável crescimento na área florestal pelo seu aumento de área destinada a plantios florestais (Seplan, 2007).

O volume constitui uma das informações de maior importância para o conhecimento do potencial disponível em um povoamento florestal, haja vista que o volume individual fornece subsídios para a avaliação do estoque de madeira e análise do potencial produtivo das florestas (Thomas et al., 2006). Para gerar estimativas precisas desta variável são utilizados os modelos de regressão. Podem ser realizados através da cubagem rigorosa, que se obtém o volume real. Na cubagem rigorosa, a árvore é dividida em seções, de comprimentos iguais ou não; por meio da utilização de fórmulas geométricas o volume dessas seções é obtido e, por adição destas partes, o volume da árvore é calculado (FAO, 1973). É importante cubar um número mínimo de árvores para caracterizar a variância dentro de cada classe diamétrica (Soares et al., 2006).

Neste sentido, o ajuste de equações volumétricas para povoamentos de Calophyllum brasiliense Cambess por meio da cubagem rigorosa busca proporcionar estimativas precisas do volume sem a necessidade de derrubada dos indivíduos no futuro, reduzindo assim o custo operacional do inventário florestal. E para isso o teste com as oito equações volumétricas será calculado, para se analisar qual equação apresentara o menor erro e maior aproximação do volume estimado da área.

\section{MATERIAL E MÉTODOS}

$\mathrm{O}$ estudo foi realizado em um plantio comercial da espécie Calophyllum brasiliense Cambess (Guanandi) aos com 7 anos de idade, de propriedade da empresa JAMP Agropecuária e Reflorestadora Ltda. (Figura 1), situada no município de Dueré, região sul do estado do Tocantins.

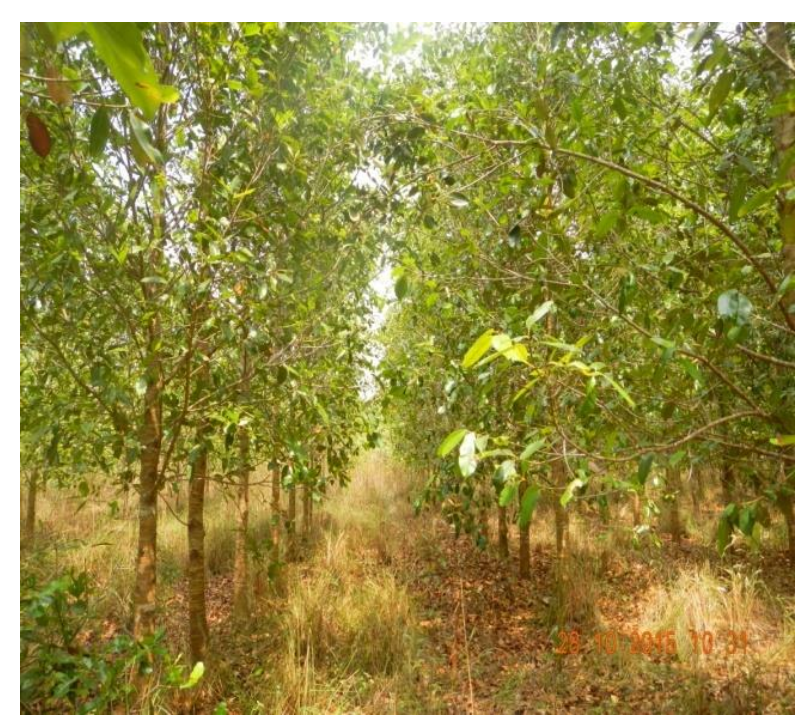

Figura 1 - Plantio comercial de Calophyllum brasiliense Cambess.

A fazenda Reunida, de propriedade da empresa JAMP Agropecuária e Reflorestadora Ltda., onde está implantado o plantio comercial está situada a 225 metros de altitude, entre as coordenadas geográficas latitude: $11^{\circ} 20^{\prime} 46^{\prime \prime}$ sul e longitude: 49 $16^{\prime} 6^{\prime}$ " oeste (Figura 2), distante $221 \mathrm{~km}$ da capital Palmas (Tocantins).

A propriedade conta com uma superfície total de 25 mil hectares, sendo as atividades voltadas para lavoura, pecuária e plantios de espécies florestais (Eucalipto e Guanandi). O Guanandi é plantado em duas áreas, sendo uma com 35 ha e outra com 970 hectares. 

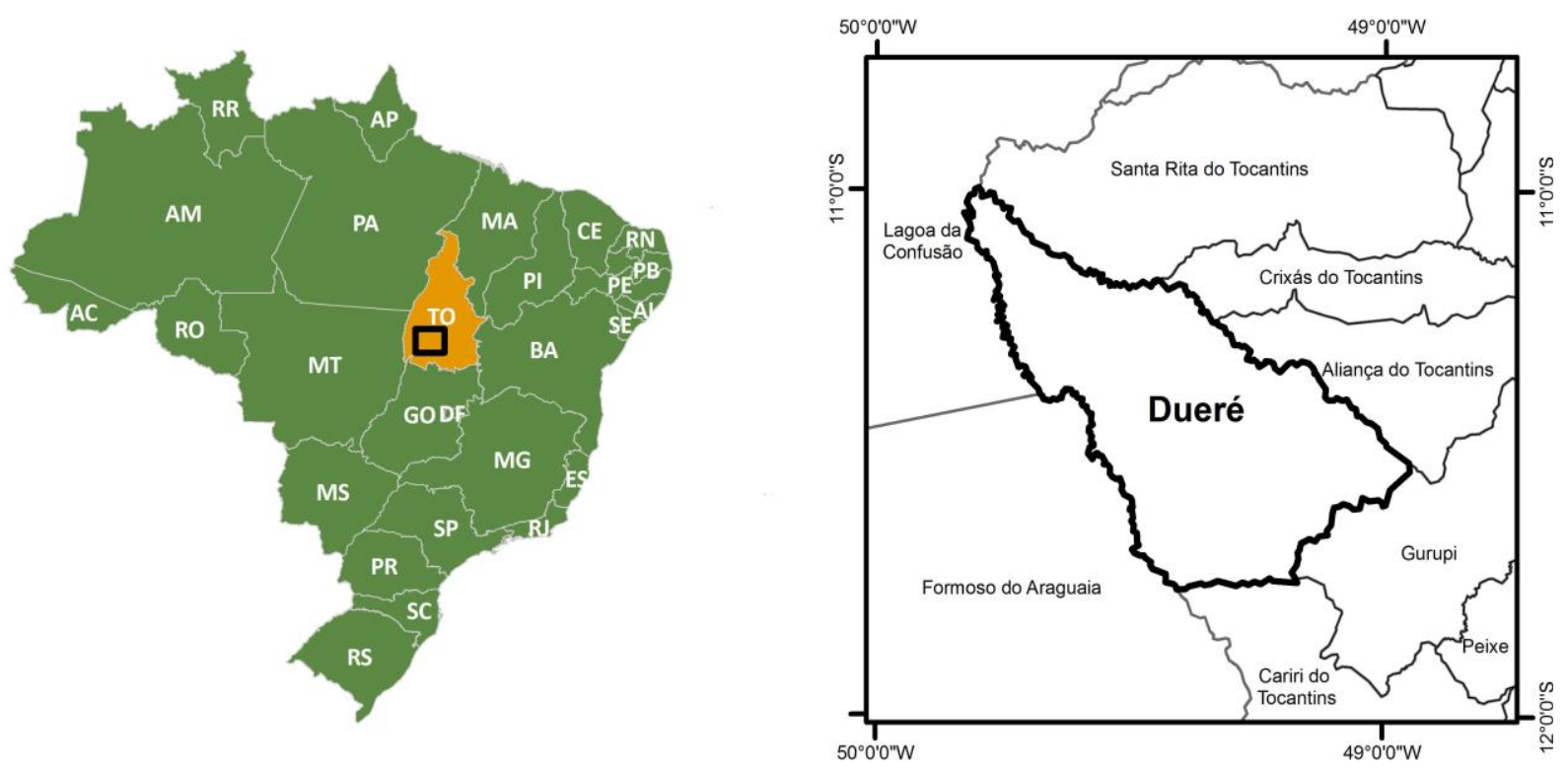

Figura 2 - Localização geográfica da área de estudo destacando o estado do Tocantins e o município de Dueré.

O solo da região de estudo é classificado como Latossolos e compreende áreas formadas por solos variando entre bem a fortemente drenados. São solos profundos e ocorrem em relevo suave ondulado (predomínio de declives igual ou inferior a 5\%). Os processos de escoamento superficial são difusos e lentos, com eventuais escoamentos concentrados (Sano et al., 2008).

\section{Cubagem rigorosa}

Foi realizada a cubagem pelo método de Smalian, onde o volume de cada secção é calculado em função do comprimento e das áreas basais nas extremidades das secções.

Para definir quantas árvores deveriam ser cubadas rigorosamente, foi necessário estabelecer uma amostra piloto, esta amostra consistiu na cubagem de pequeno número de árvores, de tal forma, que se tenha ideia da variabilidade entre os volumes das árvores que compõem a população considerada (Mello, 2004).

Seguindo este preceito foram cubadas 46 árvores-amostra para determinação do volume rigoroso utilizando a metodologia de Smalian, que indica que o volume de cada seção foi somado para obter o volume total das toras de acordo com a seguinte expressão proposta por Finger (1992) como mostrado na equação 1 .

$$
v=v_{0}+\sum_{i=1}^{n} v_{i}+v_{c} \quad(\text { Equação } 1)
$$

Em que: $v=$ volume, $v_{0}=$ volume do toco, $v_{i}=$ volume das secções transversais e $v_{c}=$ volume do cone.
A seleção das árvores para a cubagem foi realizada a partir da distribuição da frequência diamétrica. Foi feita a seleção e a cubagem rigorosa de árvores-amostra abatidas com CAP $\geq 15 \mathrm{~cm}$ (DAP $\geq 4 \mathrm{~cm}$ aproximado) de forma a contemplar toda a distribuição diamétrica encontrada na área inventariada aos 7 anos de idade.

O número de árvores a ser cubadas foi definido através da equação 2 descrita por Mello (2004):

$$
n=\frac{t^{2} \cdot(C V \%)^{2}}{E^{2} \%}
$$

Em que: $\mathrm{t}=$ valor tabelado (Student) $\mathrm{CV} \%=\mathrm{co}-$ eficiente de variação; $E$ = erro pré-estabelecido, neste caso utilizado $10 \%, \mathrm{n}=$ número de árvores a serem cubadas rigorosamente.

\section{Determinação da equação volumétrica}

Com os dados obtidos na cubagem foram calculados os volumes individuais para cada uma das árvores-amostra. $\mathrm{O}$ fator de forma artificial foi obtido pela razão entre o volume rigoroso e o volume do cilindro com diâmetro $1,3 \mathrm{~m}$ de acordo com a equação 3 (Finger, 1992):

$$
f_{1,30}=\frac{\text { volume rigoroso }}{\text { volume do cilindro }}
$$

(Equação 3)

Em que: $f_{1,30}=$ fator de forma artificial; $d_{1,3}=$ diâmetro medido a $1,30 \mathrm{~m}$ da altura total da árvore.

Os dados destas árvores serviram de base para o ajuste dos modelos de volume relacionados na tabela 1. 
Tabela 1 - Modelos de equações para determinação do volume individual em metros cúbicos do plantio comercial de Calophyllum brasiliense Cambess em Dueré- TO.

\begin{tabular}{c|c|l}
\hline $\mathbf{N}^{\mathbf{0}}$ & Função & \multicolumn{1}{c}{ Modelo } \\
\hline 1 & Hohenald-Krenn & $v=\beta_{0}+\beta_{1} \cdot d+B_{2} \cdot \mathrm{d}^{2}$ \\
2 & Spurr & $v=\beta_{0}+\beta_{1} \cdot d^{2} \cdot h$ \\
3 & Stoate & $v=\beta_{0}+\beta_{1} \cdot d^{2}+\beta_{2} \cdot\left(d^{2} \cdot \mathrm{h}\right)+\beta_{3} \cdot \mathrm{h}$ \\
4 & Näslund & $v=\beta_{1} \cdot d^{2}+\beta_{2} \cdot\left(d^{2} \cdot \mathrm{h}\right)+\beta_{3} \cdot\left(d \cdot \mathrm{h}^{2}\right)+\beta_{4} \cdot \mathrm{h}^{2}$ \\
5 & Meyer & $v=\beta_{0}+\beta_{1} \cdot d+\beta_{2} \cdot d^{2}+\beta_{3} \cdot(d \cdot \mathrm{h})+\beta_{4} \cdot\left(d^{2} \cdot \mathrm{h}\right)$ \\
6 & Meyer (modificado) & $v=\beta_{0}+\beta_{1} \cdot d+\beta_{2} \cdot d^{2}+\beta_{3} \cdot(d \cdot \mathrm{h})+\beta_{4} \cdot\left(d^{2} \cdot \mathrm{h}\right)+\beta_{5} \cdot \mathrm{h}$ \\
7 & Spurr & $\ln v=\beta_{0}+\beta_{1} \cdot \ln \left(d^{2} \cdot \mathrm{h}\right)$ \\
8 & Schumacher-Hall & $\ln v=\beta_{0}+\beta_{1} \cdot \ln d+\beta_{2} \cdot \ln \mathrm{h}$ \\
\hline
\end{tabular}

Em que: $v=$ volume individual em $\mathrm{m}^{3} ; \mathrm{h}=$ altura total $(\mathrm{m}) \mathrm{e} \mathrm{d}=$ diâmetro a altura do peito em $\mathrm{cm} ; \beta_{0}, \beta_{1}, \beta_{2}, \beta_{3}, \beta_{4} e \beta_{5}=$ coeficientes da regressão. Fonte: Prodan (1968); Schneider (1984).

\section{Seleção dos modelos matemáticos}

Para a seleção dos modelos matemáticos foram considerados alguns parâmetros estatísticos, sendo eles: Coeficiente de determinação ajustado ( $\left.R^{2} a j\right)$, Coeficiente de variação em porcentagem (CV\%), e análise gráfica de resíduos.

O coeficiente de determinação ajustado expressa a quantidade da variação explicada pela regressão considerando o número de coeficientes da equação e selecionando o modelo matemático que apresente maior valor de $\mathrm{R}^{2}$ aj, obtido pela equação 4 (Schneider e Schneider, 2008):

$$
\mathrm{R}_{\mathrm{aj}}^{2}=\mathrm{R}^{2}-\left[\frac{\mathrm{K}-1}{\mathrm{~N}-\mathrm{K}}\right] \cdot\left(1-\mathrm{R}^{2}\right) \quad(\text { Equação } 4)
$$

Em que: $\mathrm{R}_{\mathrm{aj}}^{2}=$ coeficiente de determinação ajustado; $R^{2}=$ coeficiente de determinação; $K=$ número de coeficientes da equação; $\mathrm{N}=$ número de observações.

O coeficiente de variação mede a dispersão relativa entre os valores observados e estimados pela regressão e nesse sentido, permitiu selecionar o modelo matemático que apresentou menor valor de CV (\%). Esse coeficiente é expresso pela equação 5 (Draper et al.,1998):

$$
C V \%=\frac{s_{y x}}{\hat{y}} \cdot 100 \quad(\text { Equação 5) }
$$

Em que: $S_{y x}=$ erro padrão da estimativa; $C V \%=$ coeficiente de variação em percentagem; $\hat{y}=$ média aritmética da variável dependente.

A análise gráfica de resíduos é feita entre as variáveis dependentes observadas e estimadas. Esta análise permite detectar possíveis tendências de ajuste ao longo da linha da regressão para, assim, determinar qual modelo seria selecionado como o melhor (Menon, 2005).

Para verificar a precisão da equação ajustada e a existência de estimativas tendenciosas foi feita a análise gráfica dos resíduos. A tendência de superestimar ou subestimar as estimativas pode ser observado na distribuição não uniforme dos dados ao longo da reta que correspondeu ao resíduo zero (Menon, 2005), sendo expressa pela equação 6:

$$
E_{i}=\left(\frac{Y_{i}-\hat{Y}_{i}}{Y_{i}}\right) \cdot 100 \quad \text { (Equação 6) }
$$

Em que: $E_{i}=$ resíduo da i-ésima observação; $Y_{i}$ = valor real da árvore; $\widehat{Y}_{i}=$ valor estimado da árvore.

\section{RESULTADOS E DISCUSSÃO}

O número de árvores cubadas por classe diamétrica foi proporcional à distribuição de frequência do povoamento por classe variando de 15 a $20 \%$ para que pelo menos um indivíduo por classe fosse amostrado. Esta metodologia também foi utilizada por Miranda et al. (2014) que ao avaliarem o efeito do método de amostragem da cubagem rigorosa na precisão de estimativas volumétricas em plantios clonais de Eucalyptus grandis W. Hill ex Maiden $\mathrm{x}$ Eucalyptus urophylla S. T. Blake, localizados na Bahia, com idade variando de 58 a 65 meses, ajustaram o modelo de Schumacher-Hall a duas bases de dados, sendo a primeira com número fixo de árvores em todas as classes diamétricas e a segunda proporcional à distribuição diamétrica do povoamento. Os resultados demonstraram que a maior precisão foi obtida com a amostragem proporcional, o que permite redução do tempo e dos custos 
da operação de cubagem rigorosa.

Tabela 2 - Frequência absoluta das árvores cubadas para o plantio comercial de Calophyllum brasiliense Cambess Em Dueré-TO para classes de DAP com amplitude de $2 \mathrm{~cm}$.

\begin{tabular}{cc}
\hline Classes DAP $(\mathbf{c m})$ & $\begin{array}{c}\text { Número de árvores } \\
\text { cubadas }\end{array}$ \\
\hline $4-6$ & 01 \\
$6-8$ & 16 \\
$8-10$ & 11 \\
$10-12$ & 05 \\
$12-14$ & 12 \\
$14-16$ & 01 \\
\hline TOTAL & 46 \\
\hline
\end{tabular}

De posse dos resultados volumétricos das 46 árvores cubadas rigorosamente pelo método de Smalian, procedeu-se com o cálculo correspondente de aplicação dos oito modelos volumétricos descritos na Tabela 1. Os coeficientes de determinação ajustados variaram entre 0,92 e 0,98 e coeficiente de variação entre 2,2 a $14,7 \%$ como mostrado na tabela 3 .

Tabela 3 - Coeficiente de determinação ajustado e coeficiente de variação para os modelos volumétricos avaliados.

\begin{tabular}{l|cc}
\hline \multicolumn{1}{c|}{ Função } & $\mathbf{R}^{\mathbf{2}}$ aj & CV\% \\
\hline Hohenald-Krenn & 0,922 & 14,69 \\
Spurr & 0,963 & 9,99 \\
Stoate & 0,975 & 8,22 \\
Näslund & 0,976 & 8,03 \\
Meyer & 0,975 & 8,27 \\
Meyer modificado & 0,974 & 8,38 \\
Spurr log & 0,974 & 2,51 \\
Schumacher-Hall & 0,979 & 2,24 \\
\hline
\end{tabular}

Em que: $\mathrm{R}^{2}$ aj $=$ Coeficiente de determinação ajustado; $\mathrm{CV} \%=$ coeficiente de variação em porcentagem.

Os modelos foram significativos ao nível de probabilidade estabelecida e, portanto, conclui-se que pelo menos uma variável independente esteja relacionada com o valor da variável dependente (Tabela 4).

Tabela 4 - Parâmetros dos modelos volumétricos avaliados.

\begin{tabular}{l|cccccc}
\hline \multicolumn{1}{c}{ Função } & $\boldsymbol{\beta}_{\mathbf{0}}$ & $\boldsymbol{\beta}_{\mathbf{1}}$ & $\boldsymbol{\beta}_{\mathbf{2}}$ & $\boldsymbol{\beta}_{\mathbf{3}}$ & $\boldsymbol{\beta}_{\mathbf{4}}$ & $\boldsymbol{\beta}_{\mathbf{5}}$ \\
\hline Hohenald-Krenn & $-0,01846^{*}$ & $3,85 \mathrm{E}^{-05 *}$ & $1,03 \mathrm{E}^{-08 *}$ & - & - & - \\
Spurr & $0,00526^{*}$ & $3,09068 \mathrm{E}^{-09 *}$ & - & - & - & - \\
Stoate & $-0,00935^{*}$ & $2,33438 \mathrm{E}^{-10 *}$ & $2,3537 \mathrm{E}^{-09 *}$ & $0,002808776^{*}$ & - & - \\
Näslund & $-0,00339^{*}$ & $8,27 \mathrm{E}^{-09}$ & $1,32 \mathrm{E}^{-09 *}$ & $-9,7 \mathrm{E}^{-07 *}$ & $0,000276^{*}$ & - \\
Meyer & $0,01646 *$ & $-6,4 \mathrm{E}^{-05 *}$ & $3,55 \mathrm{E}^{-08 *}$ & $7,24 \mathrm{E}^{-06 *}$ & $-1,7 \mathrm{E}^{-09 *}$ & - \\
Meyer modificado & $0,01106^{*}$ & $-5,2 \mathrm{E}^{-05 *}$ & $3 \mathrm{E}^{-08 *}$ & $5,45481 \mathrm{E}^{-06 *}$ & $-8,54597 \mathrm{E}^{-10 *}$ & $0,000858312^{*}$ \\
Spurr log & $-16,4101^{*}$ & $0,813535^{*}$ & - & - & - & - \\
Schumacher-Hall & $-15,0053^{*}$ & $1,29298^{*}$ & $1,266519 *$ & - & - & - \\
\hline
\end{tabular}

Em que: $\beta_{0}, \beta_{1}, \beta_{2}, \beta_{3}, \beta_{4} e \beta_{5}=$ parâmetros das equações.

A análise gráfica de dispersão de resíduos (Figura 3), entre os valores observados e estimados, mostrou que a função $v=-15,0053+$ $1,29298 \cdot \ln (d)+1,266519 \cdot \ln (h)$ (SchumacherHall) apresentou ajuste mais adequado para o conjunto de dados entre as equações testadas, evidenciando a seleção do modelo.

O modelo volumétrico de Schumacher-Hall é muito usado para determinar o volume individual de plantios comerciais de espécies florestais, principalmente do gênero Eucalyptus e Pinus.

Neste sentido Rocha et al. (2010) ao avaliarem métodos de estimativas de volume para povoamento de Eucalyptus urophylla com 57 meses de idade no planalto da Conquista (BA) determinaram que a partir do inventário tradicional, o modelo volumétrico que apresentou o melhor desempenho foi o de Schumacher-Hall (logarítmica). A dispersão gráfica dos resíduos não apresentou tendenciosidade, com um $R^{2} a j=0,95$, também Azevedo et al. (2011a) ao determinarem estimativas volumétricas para Eucalyptus aos cinco anos de idade sob regime de alto fuste e talhadia no sudoeste da Bahia selecionaram o modelo de Schumacher-Hall logaritmizado com $\mathrm{R}^{2}$ aj de 0,98 e CV\% de 4,66 para volume total.

Já para a espécie Calophyllum brasiliense, Silva et al. (2019) encontrou um R ${ }^{2}$ aj de 0,979 e CV de $7,5 \%$ ao usar o modelo de Schumacher e Hall para estimar o volume total, além de testar modelos de funções probabilísticas para o Guanandi aos 94 meses na região sul do Tocantins, mostrando que o modelo pode ser usado com a espécie na região estudada. 


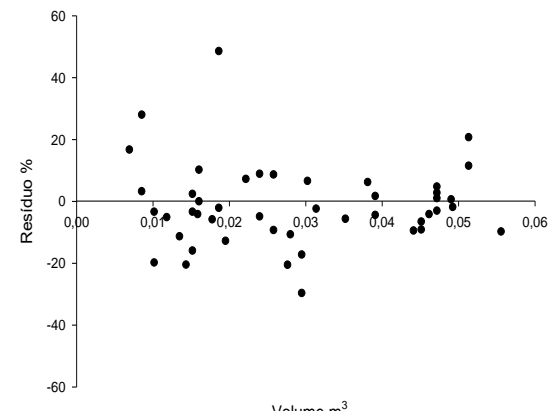

Hohenald

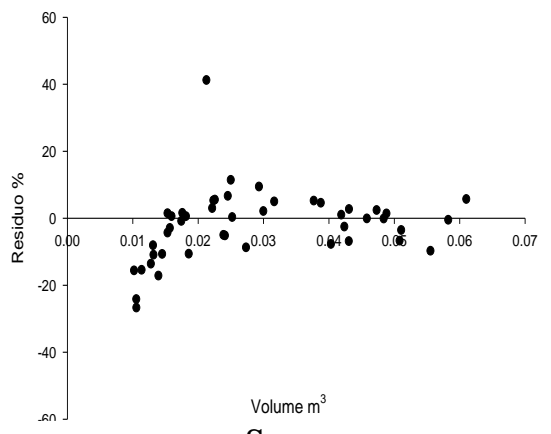

Spurr

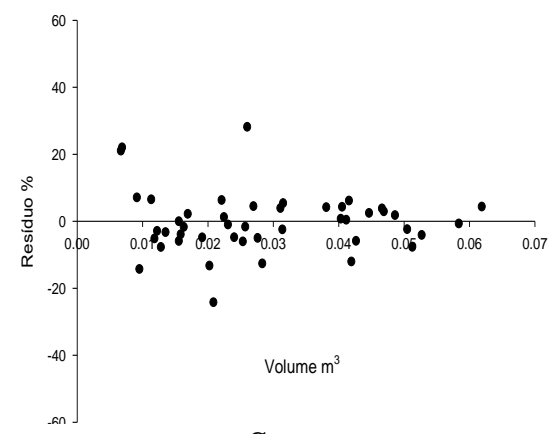

Stoate

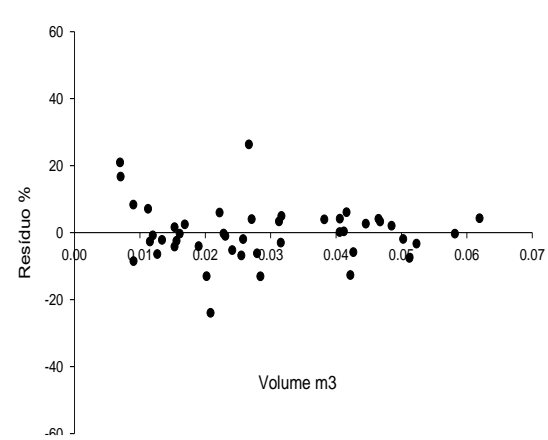

Näslund

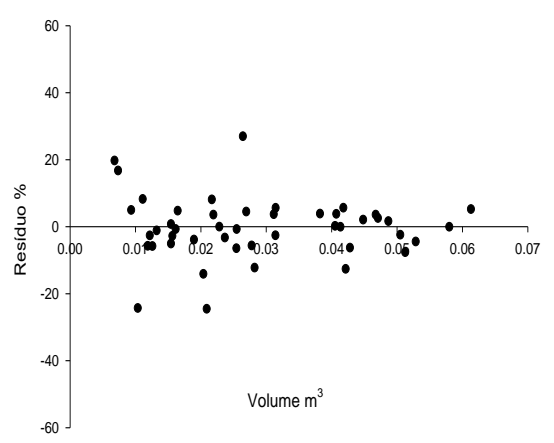

Meyer

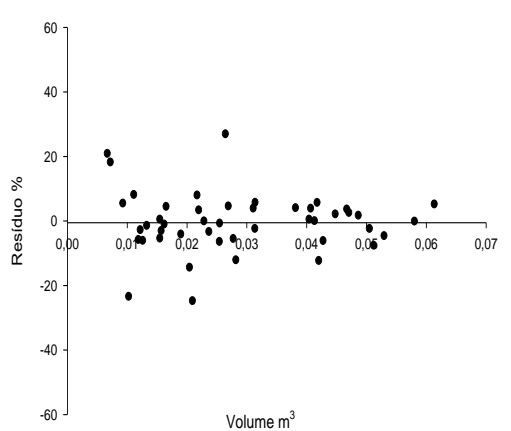

Meyer Modificado

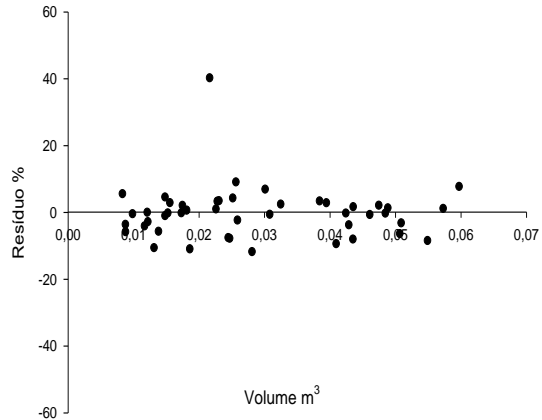

Spurr log

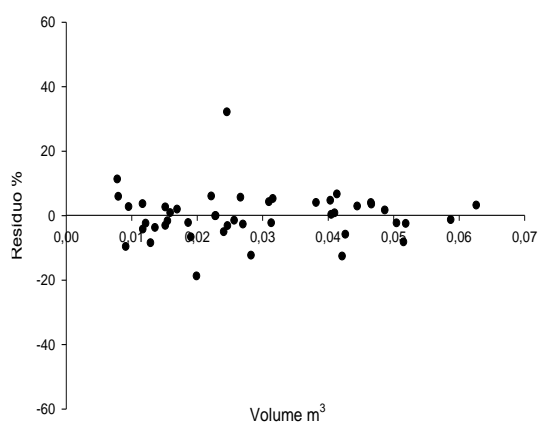

Schumacher-Hall

Figura 3 - Análise gráfica de resíduo para os modelos volumétricos avaliados.

\section{CONCLUSÃO}

O modelo volumétrico de Schumacher-Hall descrito pela função $\ln (v)=-15,0053+$ 1.29298. $\ln (d)+1,266519$. $\ln (h)$ apresentou o melhor ajuste e elevada precisão estatística para o conjunto de dados analisados, evidenciando que este modelo poderá ser usado como suporte aos correspondentes planos de manejo de reflorestamento de Calophyllum brasiliense Cambess no Tocantins.

\section{REFERÊNCIAS BIBLIOGRÁFICAS}

AZEVEDO, G.B.; SOUSA, G.T.O.; BARRETO, P.A.B.; CONCEICAO JÚNIOR, V. Métodos para predição do volume de eucalipto em diferentes regimes de manejo no Planalto da Conquista. In: II Semana de Engenharia Florestal da Bahia (2 ${ }^{\text {a }}$ SEEFLOR-BAHIA), 2011, Vitória da Conquista-BA. Anais..., Vitória da Conquista, 2011a.

CARVALHO, P. E. R. Influência da intensidade luminosa e do substrato no crescimento, no conteúdo de clorofila e na fotossíntese de Cabralea canjerana (Vell.) Mart. subsp. canjerana, Calophyllum brasiliense Cambess. Camb. e Centrolobium robustum (Vell.) Mart. ex Benth., na fase juvenil. Tese Doutorado. Curitiba: Universidade Federal do Paraná, 157p. 1996.

CIDRÃO, D.F. Economia florestal: potencialidades do Guanandi. 2012. 184 f. Dissertação (Mestrado) - UNIARA, Araraquara, 2012.

DRAPER, N.R.; SMITH, H. Applied regression analysis. 3rd ed. New York: John Wiley \& Son, 1998. xvii, 706p.

DUFFECKY, M.D.; FOSSATI, L.C. Avaliação da adaptação de Calophyllum brasiliense Cambess. (Guanandi), família 
Clusiaceae, no planalto norte catarinense. Ágora: R. Divulg. Cient., Mafra, v.16, n.2, 2009.

FAO. Manual of forest inventory, with special reference to mixed tropical forest. Roma, 1973. 200p.

FINGER, C.A.G. Fundamentos de biometria florestal. Santa Maria: UFSM, CEPEFFATEC, 1992.

KÖPPEN, W.; GEIGER, R. Klimate der Erde. Gotha: Verlag Justus Perthes. 1928.

MELLO, J. M de. Geoestatística aplicada ao inventário florestal (Tese de Doutorado). Piracicaba: Doutorado em Recursos florestais, Escola Superior de Agricultura Luiz de Queiroz. Universidade de São Paulo. 2004

MENON, M. U. Ajuste dos modelos de Kozak e do sistema Murkhart e Cao para plantações de Pinus oocarpa. Revista Ambiência, Guarapuava, PR, n.1, p.197-205, 2005.

MIRANDA, R.O.V.; EBLING, A.A.; DAVID, C.H.; FIORENTIN, L.D.; SOARES, I.D. Efeito do método de amostragem da cubagem rigorosa na precisão de estimativas volumétricas. Atualidades em Mensuração Florestal. Curitiba - Paraná - Brasil. 2014.

PRODAN, M. Forest biometrics. New York: Pergamon Press, 1968. 447p.

ROCHA, T.B., CABACINHA, C.D., ALMEIDA, R.C; PAULA, A.; SANTOS, R.C. Avaliação de métodos de estimativa de volume para um povoamento de Eucalyptus urophylla S. T. Blake no Planalto da Conquista - BA. Enciclopédia Biosfera, Centro Científico Conhecer - Goiânia, v.6, n.10, 2010.

SANO, E.E.; ROSA, R.; BRITO, J.L.; FERREIRA J.R.L.G. 2008. Mapeamento semidetalhado do uso da terra do bioma Cerrado. Planaltina, Pesquisa Agropecuária Brasileira, v.43, p.153-156.

SCHNEIDER, P.R. ANATRO: Programa de computador ambiente MS-DOS. Santa Maria: Universidade Federal de Santa Maria, 1984.

SCHNEIDER, P.R.; SCHNEIDER, P.S. Introdução ao manejo florestal. $2^{\circ}$ Ed. Santa Maria: FACOS-UFSM, 2008, 566p.

SEPLAN - Secretária do Planejamento e Meio Ambiente. Anuário estatístico do estado do Tocantins. Palmas: Diretoria de Pesquisas e Informações-DPI, 2007.

SILVA, M.C.; VIEIRA, A.C.; ATAIIDE, Y.B.; RAMOS, Y.A.; COELHO, M.C.B.; GIONGO, M.; ERPEN, M.L. Volume, funções probabilísticas e produtividade em plantio de Calophyllum brasiliense no município de Dueré (TO). Advances in Forestry Science, v.6, n.2, p.631-638, 2019. http://dx.doi.org/10.34062/afs.v6i2.7400

SOARES, C.P.B.; NETO, F.P.; SOUZA, A.L. Dendrometria e inventário florestal. Viçosa: UFV, 2006. 276p.

THOMAS, C.; ANDRADE, C.M.; SCHNEIDER, P.R.; FINGER, C.A.G. Comparação de equações volumétricas ajustadas com dados de cubagem e análise de tronco. Ciência Florestal, Santa Maria, v.16, n.3, p.319-327, 2006. 